\title{
A randomized controlled trial of quetiapine for psychosis in Parkinson's disease
}

This article was published in the following Dove Press journal:

Neuropsychiatric Disease and Treatment

22 May 2009

Number of times this article has been viewed

\section{Paul Shotbolt ${ }^{1}$ \\ Michael Samuel ${ }^{2,3}$ \\ Chris Fox ${ }^{3,4}$ \\ Anthony S David'}

'Section of Cognitive

Neuropsychiatry, Institute

of Psychiatry, King's College, London,

UK; ' ${ }^{2}$ Department of Neurology, King's

College Hospital, London, UK; ${ }^{3}$ East

Kent Hospitals NHS Trust, William

Harvey Hospital, Ashford, Kent, UK;

${ }^{4}$ Kent and Medway NHS and Social

Care and Partnership Trust, Kent, UK
Introduction: Psychosis (delusions and/or hallucinations) is a well-recognized complication of treatment of Parkinson's disease (PD). Quetiapine is a currently favored treatment, but data on its efficacy are equivocal. This trial aimed to provide further evidence on the efficacy of quetiapine in PD psychosis.

Methods: We conducted a 12 week double blind randomized placebo-controlled trial. Time to dropout due to lack of improvement of psychosis was the primary outcome measure. Other important secondary outcomes were evaluated using standard rating scales for PD and psychiatric symptoms.

Results: Twenty-four eligible subjects gave consent. The primary outcome, time to dropout, was examined using survival analysis. It was shown that patients in the quetiapine group dropped out earlier than those in the placebo group, but this difference was not significant $(p=0.68)$. No significant changes were found for any of the secondary outcome measures in either group.

Conclusions: In this study, quetiapine at doses of up to $150 \mathrm{mg} /$ day failed to significantly improve psychosis compared to placebo, however the small sample size does not allow any conclusive interpretation of the results. Quetiapine did not appear to worsen PD motor functioning, but its use was limited by a faster drop out compared with placebo. Significant impediments were difficulty with recruitment and natural fluctuation in symptoms during the trial.

Keywords: Parkinson's disease, psychosis, antipsychotics, quetiapine

\section{Introduction}

Psychosis (delusions and/or hallucinations) is a well-recognized complication of treatment of Parkinson's disease (PD). A literature review gave the prevalence of hallucinations in clinic populations as between $8 \%$ and $44 \% .{ }^{1}$ Risk factors for psychosis include older age, longer duration of disease, cognitive impairment, and sleep disturbance. ${ }^{1,2}$ Psychosis in PD is an indicator of poor prognosis and is frequently the deciding factor in necessitating nursing/institutional care. ${ }^{3,4}$

Until recently, managing psychosis in patients with PD posed clinicians with the "motion-emotion" dilemma: attempts to alleviate the psychosis, either by reducing antiparkisonian medication or introducing antipsychotic agents, led to worsening of the parkinsonian motor symptoms. ${ }^{5}$ The introduction of 'atypical' antipsychotic drugs into psychiatric clinical practice opened up a new range of therapeutic options. The most commonly used include clozapine, risperidone, olanzapine, and quetiapine. All have been used to treat psychosis in PD with varying success. ${ }^{6}$ Clozapine has been the most frequently evaluated to date with over 30 published reports. There have been two randomized placebo-controlled trials. ${ }^{7,8}$ Both studies showed significant
Correspondence: Paul Shotbolt PO68, The Institute of Psychiatry, De Crespigny Park, Denmark Hill, London SE5 8AF, UK

Tel +442084442I35

Fax +442078480572

Email p.shotbolt@imperial.ac.uk 
improvements in psychopathology with no or minimal worsening in motor symptoms of PD. The main drawback of clozapine is the need for regular blood monitoring due to the risk of agranulocytosis (up to $1 \%$ ). Initial reports of efficacy of risperidone and olanzapine were encouraging but subsequently these drugs were shown to worsen parkinsonian motor function..$^{9,10}$

Quetiapine is one of the currently favored atypical antipsychotic treatments. It produces few parkinsonian side effects in patients with bipolar disorder and minimal increases in prolactin in patients with schizophrenia. ${ }^{11,12}$ A systematic review revealed 10 reports of quetiapine use in PD psychosis involving 242 patients. ${ }^{13}$ Of these 191 (80\%) were claimed to show improvement in psychosis and only $25(11 \%)$ worsening of motor symptoms. The most recent randomized placebo-controlled trials found no benefit of quetiapine. ${ }^{14-16}$

In an alternative approach, two small unblinded studies have directly compared quetiapine with the best alternative, clozapine. The first found both drugs to be equally efficacious. ${ }^{17}$ The other found clozapine had a significant advantage over quetiapine in reducing delusions, but showed only a nonsignificant trend over quetiapine in controlling the frequency of hallucinations. ${ }^{18}$ In addition one patient in the clozapine arm developed leukopenia. These data provide an incomplete and sometimes conflicting picture. This doubleblind randomized controlled trial attempted to provide further evidence on the efficacy of quetiapine in the management of PD psychosis.

\section{Methods}

We conducted a 12 week randomized, placebo-controlled double-blind clinical trial of quetiapine in PD psychosis. All patients met UK Brain Bank criteria for idiopathic PD. They were included if they suffered from either 'hallucinations', 'suspiciousness', or 'unusual thought content (delusions)' of a severity $>3 / 7$, on the Brief Psychiatric Rating Scale (BPRS). ${ }^{19}$ Symptoms must have been present for over two weeks. Other inclusion criteria included: a reliable caregiver; ability to assent to treatment; current antiparkinsonian treatment deemed to be optimal by the attending specialist consultants and communication ability sufficient to enable main assessments. Patients were recruited from the King's College Hospital Regional Neurosciences Centre Movement Disorder Clinic and outlying clinics led by consultant neurologists and geriatricians with specialist expertise in PD. The trial was pragmatic in the sense that clinicians had flexibility regarding the antiparkinsonian regimen used, and also there were no exclusions based on age, dementia, or disease severity provided other practical inclusion criteria were met.

Exclusion criteria included current treatment with cholinesterase inhibitors, antipsychotic medication currently or in the preceding two weeks, any contraindication to quetiapine, important drug interactions, major concomitant medical illness, stroke or transient ischemic attack in the six months preceding assessment, uncontrolled diabetes or hypertension, uncontrolled atrial fibrillation or other cardiac arrhythmia, past drug/alcohol dependence, possible delirium and change in medication over the preceding two weeks (three weeks if cabergoline). Patients who had dementia with Lewy bodies were excluded. ${ }^{20}$ The protocol was approved by the local ethics committees and all subjects gave written informed consent/assent prior to the study.

Stopping rules enabled patients and their clinicians to withdraw from the study because of nonimprovement or clinically important worsening in psychiatric symptoms, and if found to be on placebo, for them to be offered open-label quetiapine as a first choice antipsychotic. This design was employed to address the issue of administering a placebo medication to symptomatic patients.

Those remaining on treatment under double-blind conditions were followed for a total of 12 weeks. From our experience with this clinical group we anticipated that few patients would be able to remain in a long trial under doubleblind conditions. Time remaining in the trial was therefore the primary outcome. This would give a measure of treatment efficacy as the design meant that patients would drop out if their psychosis failed to improve or deteriorated and would stay in if their symptoms were improving. As this was a pragmatic trial, time to drop out due to side-effects was also included in the analysis.

The main secondary outcome was improvement in psychotic symptoms. Other important secondary outcomes were evaluated using standard rating scales; the Unified Parkinson's Disease Rating Scale (UPDRS), BPRS, Neuropsychiatric Inventory (NPI), and the Baylor PD hallucination scale. ${ }^{14,21,22}$

Power calculation was based of a $50 \%$ cessation of treatment seen in the 12 week US clozapine study. ${ }^{7}$ We assumed that $50 \%$ of the quetiapine group and $90 \%$ of the placebo group would drop out of treatment by 12 weeks, generating $87 \%$ power to detect time to drop-out as statistically different using a log-rank test with a two-sided significance level of 5\% with 30 patients per group (total $=60$ ). In the US clozapine study, the four-week mean BPRS for the placebo group was 
32.4 and for the clozapine group was 23.8. This represents a difference of 8.6 points. The pooled standard deviation was 10.3 , hence the standardized effect size is 0.835 . This could be detected with $80 \%$ power (at $5 \%$ significance level) with 24 per group in the current study.

Assessments were performed at 0, 2, 6, and 12 weeks. The study medications were randomized into quetiapine and identical appearing placebo by the manufacturers. Unblinding envelopes were held by the Clinical Trials Unit at the Institute of Psychiatry. Clinicians were free to increase or maintain dose of trial medication and identical-appearing placebo up to the beginning of the 6th week (after which it could be reduced if considered necessary due to side effects). The starting dose was $25 \mathrm{mg}$ for week 1, $25 \mathrm{mg}$ bd for week 2, $50 \mathrm{mg}$ bd for week 3, with an optional further increase to $50 \mathrm{mg}$ am, $100 \mathrm{mg}$ nocte if clinically indicated.

Survival curves (Kaplan-Meier) were plotted for both groups. In addition the time to dropout was analyzed using the log rank test. Secondary outcome measures were analyzed using analysis of covariance. The significance level was 5\% (two sided) for all analyses. Statistical analyses were performed by the Statistics unit of the Institute of Psychiatry.

\section{Results}

Sixty-seven patients were screened over a 16-month period for a target of 60 . After screening, a total of only 24 eligible subjects gave consent for enrolment. Eleven patients were randomized to quetiapine and 13 to placebo (Table 1). Thirteen patients completed six weeks in the double-blind part of the study (four quetiapine patients and nine placebo). Only eight patients completed the 12 week double blind (four from each group). Of those that dropped out, only five continued on open-label quetiapine. The mean dose in the quetiapine group was $72.7 \pm 26.1 \mathrm{mg}$; in the placebo group it was $96.2 \pm 32 \mathrm{mg}$.

Figure 1 shows the Kaplan-Meier survival functions of time to drop-out against cumulative survival. The graph shows that patients on quetiapine dropped out faster than patients on placebo. The log rank test was used to compare the survival distributions; they were not found to be significantly different $(\mathrm{p}=0.68)$. Quetiapine therefore did not have a significant effect on time to dropout.

Due to the small numbers and high drop out rate, the secondary outcome measures (Baylor PD hallucination scale, UPDRS, NPI, and BPRS) were only analyzed at six weeks. The analysis was by intention to treat and missing values were imputed using last observation carried forward. No significant changes were found for any of the secondary 


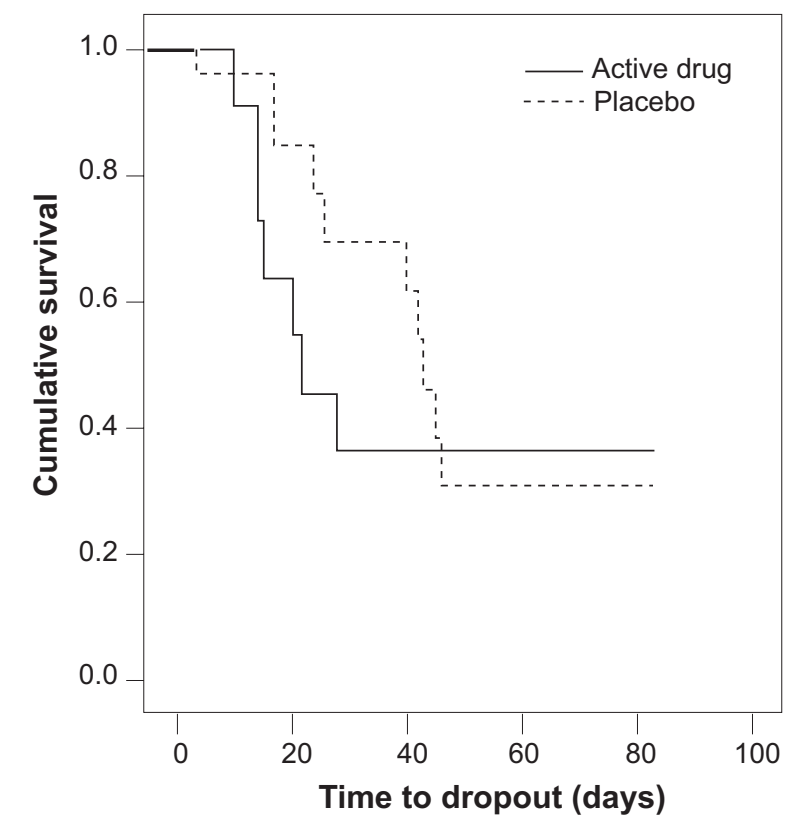

Figure I Kaplan-Meier Survival Plots (Time to dropout from trial against cumulative survival).

outcome measures in either group. Importantly there was no significant effect of quetiapine on UPDRS scores, either on total score or motor subgroup (UPDRS III). This suggests that quetiapine at the doses used in the study did not worsen motor functioning in a clinically meaningful manner.

With regard to tolerability, three patients on quetiapine dropped out due to related adverse events (drowsiness). three patients on placebo also dropped out due to related adverse events (two drowsiness, one confusion).

\section{Discussion}

In this study we were not able to show that quetiapine at doses of up to $150 \mathrm{mg}$ /day was able to significantly improve psychosis compared to placebo. Unlike some other atypical antipsychotics, quetiapine did not appear to worsen PD motor functioning. Its use was limited by a faster drop out compared with placebo, although this was not statistically significant.

Several features of the study may have limited the demonstration of a significant antipsychotic effect. Firstly, these patients with longstanding PD appeared to show considerable day-to-day fluctuation in symptom severity, based on the rater's impressions at screening and baseline visits. This made accurate measurement of baseline severity and a change in clinical condition difficult. This problem may have been compounded by the fact that we did not use a validated scale for PD psychosis as none was available at the start of the study. Both the BPRS and NPI are composite scales assessing many different neuropsychiatric symptom domains and therefore do not specifically assess psychosis. We performed separate analyses with the NPI hallucinations and delusions subscores, but there was still no difference between the two groups. The Baylor PD hallucination scale, an unvalidated scale designed for PD psychosis, revealed a trend towards improvement in a previous randomized controlled trial with quetiapine. ${ }^{14}$ However a similar trend was not seen in our study. There is now a partially validated scale for Parkinson's disease psychosis which may be a useful tool in therapeutic intervention trials, though this remains to be tested. ${ }^{24}$

Secondly the doses used in the study may not have been high enough. The mean doses used were rather low compared to other studies $\left(169 \mathrm{mg},{ }^{14} 119 \mathrm{mg},{ }^{15}\right.$ and $\left.120 \mathrm{mg}\right){ }^{16} \mathrm{It}$ is possible that of inadequate dosage contributed to the result, however the trials using higher doses also showed no benefit of quetiapine.

Thirdly despite adequate screening and adequate power to the study design, we did not recruit the necessary number of patients in the time frame required. The major weakness of our study is the small sample size, a problem shared with two of the other published randomized controlled trials. ${ }^{14,16}$ A significant factor affecting the recruitment rate was that many eligible patients referred for the study felt that their symptoms were not severe enough at the point of first interview to warrant treatment $(n=18)$. However their opinion about the severity of their symptoms was often found to be at variance with that of their carers due to impaired insight. Such cases were referred back to their consultants for observation and re-referral if their symptoms worsened.

Another reason for slow recruitment was safety concerns $(n=11)$ regarding the use of antipsychotics in the elderly with dementia. Specific data in Parkinson's disease patients are lacking, but there has been concern regarding the safety of the atypical antipsychotics in elderly patients with dementia. In 2004 the Committee on Safety of Medicines (CSM) reviewed the data for risperidone and olanzapine. ${ }^{25}$ The evidence showed a three-fold increase in the risk of stroke for risperidone when used to treat behavioral disturbance in older patients with dementia. There was a similar risk with olanzapine The US Food and Drug Administration reported a similar increased risk between 1.6 and 1.7. ${ }^{26}$ Consequently a black box warning has been added to the label of all atypical antipsychotics in the US. The CSM and FDA advice was contained in the trial patient information sheet which limited recruitment.

\section{Conclusion}

In this pragmatic trial the time to dropout was the primary outcome measure. Quetiapine did not have a significant effect on time to dropout. There was also no significant effect 
of quetiapine on any of the secondary outcome measures (BPRS, NPI, Baylor PD hallucination scale, and UPDRS). The small sample size of the study does not allow any conclusive interpretation of the results.

This trial has not answered the question of whether quetiapine is an effective treatment for PD psychosis. However, it has highlighted the difficulties in attempting to design and conduct such a placebo-controlled trial in a clinically meaningful and pragmatic manner. A significant impediment to performing the trial was the difficulty in quantifying clinically significant changes in symptom severity due to fluctuation of symptoms and the lack of a validated scale. Recruitment into the trial was slow due to safety concerns regarding the use of atypical antipsychotics in the elderly. In addition the doses of quetiapine used in the study were rather low which may have prevented any demonstration of antipsychotic effect. The effective treatment of psychosis in PD is still an urgent clinical need.

The absence of an effect of quetiapine on motor functioning was noted, as has been previously reported. ${ }^{14-16}$

\section{Acknowledgments}

We wish to thank Dr Chris Clough, Dr Robert Weeks and Professor Ray Chaudhuri, King's College Hospital; Clare Rutterford and Caroline Murphy, Institute of Psychiatry; Rosemary Vahid, Michelle McHenry (Parkinson's Disease Nurses), East Kent Hospitals NHS Trust; Dr Neil Munro, Dr Jonothan Hawkins, Dr Michael Jenkinson and Dr Andrew Heller, East Kent Hospitals Trust. The study received ethical approval from the following ethics committees; The Joint South London and Maudsley and The Institute of Psychiatry NHS Research Ethics Committee; East Kent Hospitals NHS Trust Local Research Ethics Committee; and King's College Hospital Research Ethics Committee. The study is registered with the European Clinical Trials Database. EudraCT number: 2004-002608-13. The trial was funded by the Parkinson's Disease Society. Medication was provided by AstraZeneca UK Ltd. The authors report no conflicts of interest in this work.

\section{References}

1. Barnes J, David AS. Visual hallucinations in Parkinson's disease: a review and phenomenological survey. J Neurol Neurosurg Psychiatry. 2001;70:727-733.

2. Aarsland D, Larsen JP, Cummings JL et al. Prevalence and clinical correlates of psychotic symptoms in Parkinson disease; A communitybased study. Arch Neurol. 1999;56:595-601.

3. Goetz CG, Stebbins GT. Mortality and hallucinations in nursing home patients with advanced Parkinson's disease. Neurology. 1995;45: 669-671.
4. Aarsland D, Larsen JP, Tandberg E. Predictors of nursing home placement in Parkinson's disease: a population-based, prospective study. J Am Geriatr Soc. 2000;48:938-942.

5. Cummings JL. Managing psychosis in patients with Parkinson's disease. N Engl J Med. 1999;340:801-803.

6. Factor SA, Friedman JH. Atypical antipsychotics in the treatment of drug-induced psychosis in Parkinson's disease. Mov Disord. 2000;15:201-211.

7. Low-dose clozapine for the treatment of drug-induced psychosis in Parkinson's disease. The Parkinson Study Group. N Engl J Med. 1999; 340:757-763.

8. Pollak P, Tison F, Rascol O, et al. Clozapine in drug induced psychosis in Parkinson's disease: a randomised, placebo controlled study with open follow up. J Neurol Neurosurg Psychiatry. 2004;75: 689-695.

9. Breier A, Sutton VK, Feltman PD, et al. Olanzapine in the treatment of dopamimetic-induced psychosis in patients with Parkinson's disease. Biol Psychiatry. 2002;52(5):438-445.

10. Rich SS, Friedman JH, Ott BR. Risperidone in the treatment of psychosis in six patients with Parkinson's disease and other akineticrigid syndromes. J Clin Psychiatry. 1995;56(12):556-559.

11. Nasrallah HA, Brecher M, Paulsson B. Placebo-level incidence of extrapyramidal symptoms (EPS) with quetiapine in controlled studies of patients with bipolar mania. Bipolar Disord. 2006;8: 467-474.

12. Atmaca M, Kuloglu M, Tezcan E, et al. Quetiapine is not associated with increase in prolactin secretion in contrast to haloperidol. Arch Med Res. 2002;33:562-565.

13. Reddy S, Factor SA, Molho ES, et al. The effect of quetiapine on psychosis and motor function in PD patients with and without dementia. Mov Disord. 2002;17(4):676-681.

14. Ondo WG, Tintner R, Voung KD, et al. Double-blind, placebo-controlled, unforced titration parallel trial of quetiapine for dopaminergicinduced hallucinations in Parkinson's disease. Mov Disord. 2005;20: 958-963.

15. Rabey JM, Prokhorov T, Miniovitz A, et al. Effect of quetiapine in psychotic Parkinson's disease patients: a double-blind labelled study of 3 months' duration. Mov Disord. 2007;22:313-318.

16. Kurlan R, Cummings J, Raman R, et al. Quetiapine for agitation or psychosis in patients with dementia and parkinsonism. Neurology. 2007;68:1356-1362.

17. Morgante L, Epifanio A, Spina E, et al. Quetiapine and clozapine in parkinsonian patients with dopaminergic psychosis. Clin Neuropharmacol. 2004;27:153-156.

18. Merims D, Balas M, Peretz C, et al. Rater-blinded, prospective comparison: quetiapine versus clozapine for Parkinson's disease psychosis. Clin Neuropharmacol. 2006;29:331-337.

19. Overall J, Gorham D. Brief Psychiatric Rating Scale. Psychol Rep. 1962;10:799-812.

20. McKeith IG, Golasko D, Kosaka K, et al. Consensus guidelines for the clinical and pathologic diagnosis of dementia with Lewy bodies (DLB): report of the consortium on DLB international workshop. Neurology. 1996;47:1113-1124.

21. Fahn S. Members of the UPDRS Development Committee. Unified Parkinson's Disease Rating Scale. In: Fahn S, Marsden CD, Calne DB, et al; editors. Recent developments in Parkinson's disease. 1987;11: $153-163$.

22. Cummings JL, Mega M, Gray K, et al. The Neuropsychiatric Inventory: comprehensive assessment of psychopathology in dementia. Neurology. 1994;44:2308-2314.

23. Folstein MF, Folstein SE, McHugh PR. "Mini-mental state". A practical method for grading the cognitive state of patients for the clinician. J Psychiatr Res. 1975;12:189-198.

24. Brandstaedter D, Spieker S, Ulm G, et al. Development and evaluation of the Parkinson Psychosis Questionnaire A screening-instrument for the early diagnosis of drug-induced psychosis in Parkinson's disease. J Neurol. 2005;252(9):1060-1066. 
25. Committee on Safety of Medicines. Latest news. March 9, 2004. Atypical antipsychotic drugs and stroke. Accessed on April 10, 2009. Available from: http://www.info.doh.gov.uk/doh/embroadcast.nsf/ vwDiscussionAll.
26. US Food and Drug Administration (Public Health Advisory). Deaths with antipsychotics in elderly patients with behavioural disturbances. 2005. Accessed on April 10, 2009. Available from: http://www.fda. gov/cder/drug/advisory/antipsychotics.htm.

\section{Publish your work in this journal}

Neuropsychiatric Disease and Treatment is an international, peerreviewed journal of clinical therapeutics and pharmacology focusing on concise rapid reporting of clinical or pre-clinical studies on a range of neuropsychiatric and neurological disorders. This journal is indexed on PubMed Central, the 'PsycINFO' database and CAS, and is the official

journal of The International Neuropsychiatric Association (INA). The manuscript management system is completely online and includes a very quick and fair peer-review system, which is all easy to use. Visit http://www.dovepress.com/testimonials.php to read real quotes from published authors.

Submit your manuscript here: http://www.dovepress.com/neuropsychiatric-disease-and-treatment-journal 